\title{
Les importations d'amphores Dressel 20 en Gaule Cisalpine
}

\author{
IWONA MODRZEWSKA-PiAneTti
}

\begin{abstract}
José Remesal Rodríguez examined question of Baetica olive oil imports in Germania and Rome but lacked data on Cisalpine Gaul. This study aims at supplementing the data on import of Baetica olive oil to Northern Italy. As a result of a query in deposits in Friuli and Veneto a map of distribution of amphorae type Dressel 20 on the mainland was established. The image complements data concerning Transpadana and Transalpine Gaul. A map showing finds of Baetica amphorae in the Venetian Lagoon also arose. The lagoons of the Upper Adriatic were used for a transportation of goods to Aquileia, Concordia, Altino, Oderzo, Este and Verona. Dressel 20 amphorae are most numerous in Aquileia. In remaining cities of the Adriatic coast only single pieces of amphorae for olive oil were spotted. Analysis of these amphorae deposits enabled construction of a hypothesis on the existence of microregions in imports of goods to areas of Cisalpine Gaul during the Roman period.
\end{abstract}

Keywords: Roman period, Cisalpine, Transalpine, Transpadana, olive oil imports, amphorae Dressel 20, commercial microregions

English title: Amphorae Dressel 20 Imported into the Territories of Cisalpine Gaul

Iwona Modrzewska-Pianetti, Institut d'Archéologie de l'Université de Varsovie, Warszawa; iwonamodrzewska@ poczta.onet.pl

À partir du Irr siècle av. J.-C. jusqu'au début du V' siècle ap. J.-C., Rome fut le plus grand importateur de l'huile d'olive fabriquée en Bétique. Les travaux de la mission espagnole, dirigée depuis 1989 par les professeurs José María Blázquez Martínez et José Remesal Rodríguez au Mont Testaccio à Rome, ont permis de dresser le tableau des exportations de Bétique destinées à la capitale de l'Empire (fig. 1) ${ }^{1}$. Un imposant répertoire de timbres et de tituli picti figurant sur les amphores issues de ce site est actuellement en cours d'élaboration (fig. 2). Les recherches menées par José Remesal Rodríguez ont en outre démontré

\footnotetext{
${ }^{1}$ Blázquez Martínez, Remesal Rodríguez, Rodríquez Almeida 1994.
} 


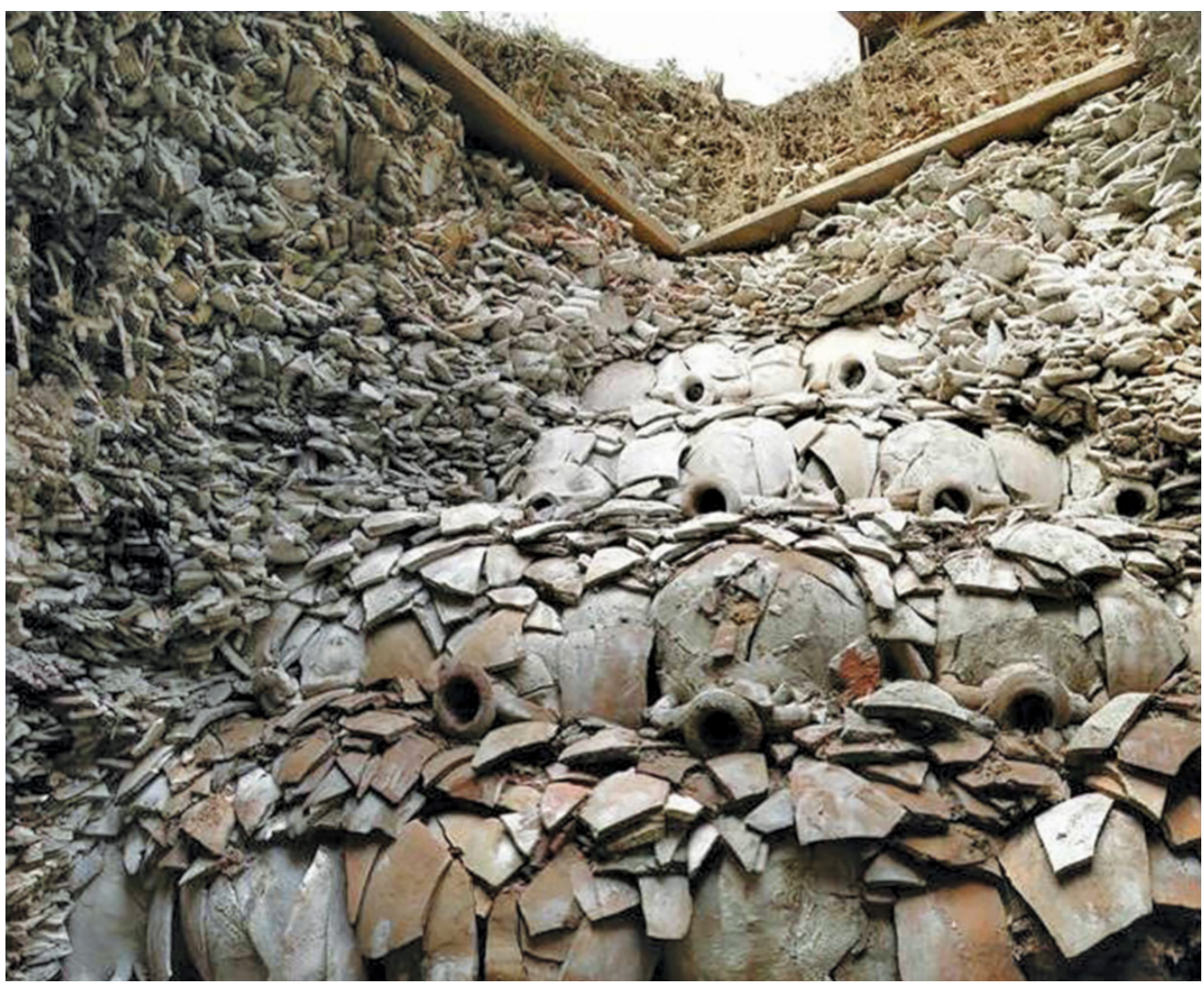

1. Le site du Mont Testaccio, tranchée avec dépôt d'amphores cassées (Archives CEIPAC, Universidad de Barcelona).

l'ampleur des importations de l'huile d'olive en Germanie ${ }^{2}$. En absence de données concernant la Gaule Cisalpine, il s'avéra opportun de lancer une étude des importations de l'huile d'olive originaire de Bétique en Italie septentrionale ${ }^{3}$.

\section{MÉTHODE D’APPROCHE}

L'élaboration de la carte de distribution des amphores Dressel 20 (Dr 20) servant au transport de l'huile d'olive a nécessité un recensement du matériel amphorique conservé dans les musées et les réserves de musées au Frioul et en Vénétie. Les données recueillies en Gaules Cisalpine, Transpadane et Transalpine ${ }^{4}$ sont venues compléter le tableau. Les recherches sur les importations de Dressel 20 en Gaule Cisalpine ont été secondées d'études comparatives concernant d'autres trouvailles d'amphores bétiques dans cette région, à savoir des Dressel 7-11, des Dressel 38 et des Beltran IIB - destinés au conditionnement des sauces

\footnotetext{
${ }^{2}$ Remesal Rodríguez 1986 ; 2002.

${ }^{3}$ Données préliminaires : Modrzewska-Pianetti 2001.

${ }^{4}$ Carre 1985 ; Brecciaroli Taborelli 1987 ; Carre, Matijaši, Pesavento Mattioli 1993 ; Tassaux 2004.
} 


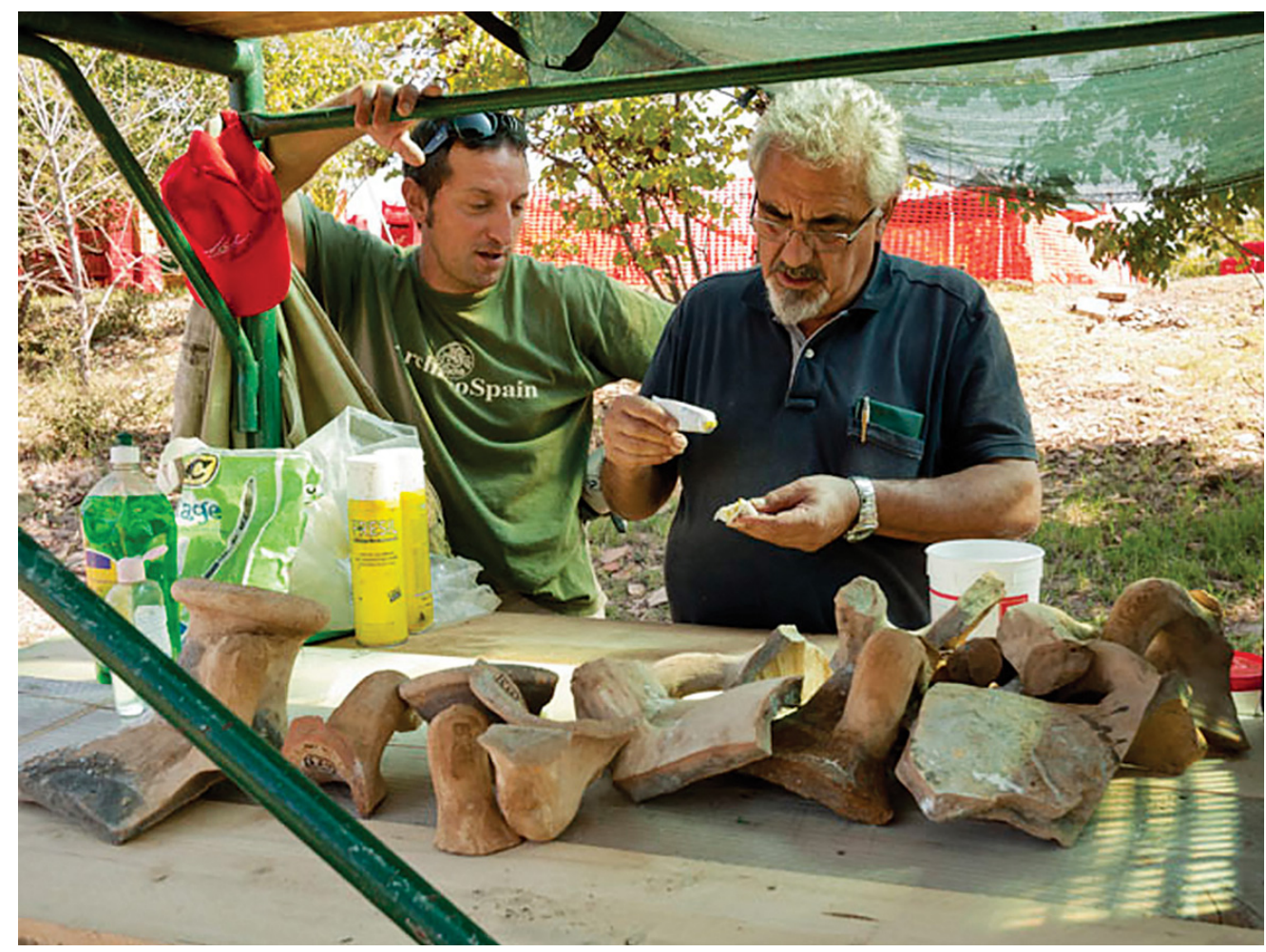

2. Le professeur José Remesal Rodríguez et dr Antonio Puig de l'Université de Barcelone en train d'identifier les timbres sur les amphores du Monte Testaccio (Archives CEIPAC, Universidad de Barcelona).

et des conserves de poisson. L'étude a porté sur le mobilier amphorique provenant des sites terrestres et des sites lagunaires (fig. 3) ${ }^{5}$. Quant à ces derniers, il s'agissait de quelques dizaines de trouvailles subaquatiques, recueillies dans le nord de la lagune de Venise. Les résultats de cette recherche ont été représentés sur des cartes de distribution des amphores dans la lagune de Venise ${ }^{6}$.

\section{L'IMPORTANCE DES LAGUNES DU HAUT ADRIATIQUE}

La lagune de Venise ainsi que d'autres lagunes du Haut Adriatique ont toujours servi à l'acheminement des produits méditerranéens vers les villes côtières, telles Aquilée, Concordia Sagittaria et Altino, d'où ils partaient par voie terrestre jusqu'à Oderzo, Padoue, Este, Vicence, Vérone et plus loin encore, dans les régions transalpines ${ }^{7}$. Ces villes et d'autres cités romaines de Gaule Cisalpine ont livré des amphores bétiques de type Dressel $20^{8}$.

\footnotetext{
${ }^{5}$ Modrzewska 1995b.

${ }^{6}$ Modrzewska 1995a: fig. 15, 52 ; Modrzewska-Pianetti 2000: fig. 40, 56.

${ }^{7}$ Carre, Matijaši, Pesavento Mattioli 1993: 390-394 ; Tassaux 2004: 168-169.

${ }^{8}$ Modrzewska-Pianetti 2001: fig. 2.
} 


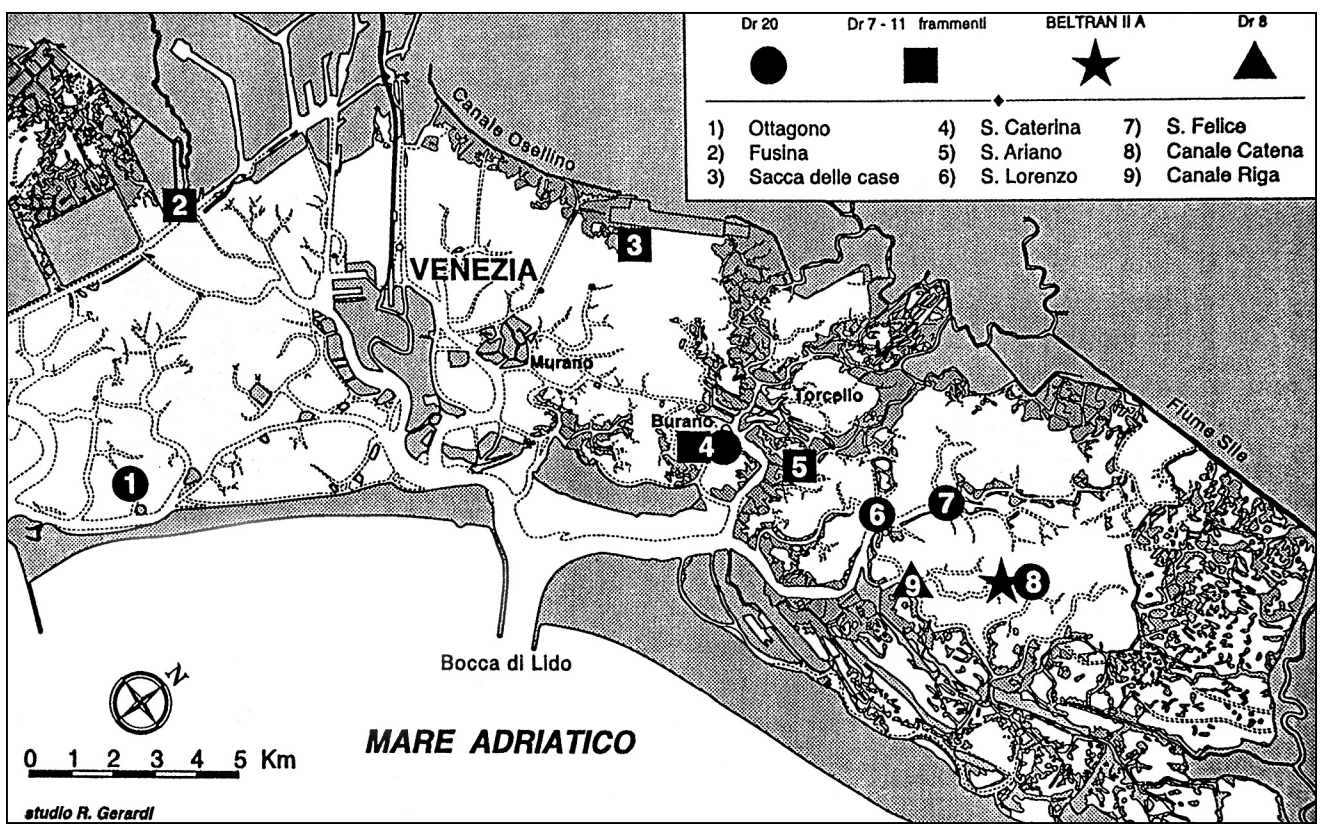

3. Lieux de trouvaille des amphores bétiques dans la lagune de Venise (plan élaboré par I. Modrzewska-Pianetti ; élaboration graphique Studio Gerardi Dolo, Vénétie ; archives de l'auteur).

Mais les amphores de ce type étaient aussi parvenues aux mansiones, à Adria et à Corte Cavanella entre autres, et à la villa romaine de San Basilio en Polésine ${ }^{9}$. Le trafic commercial passait par des ports extérieurs et intérieurs sur les fleuves débouchant dans la mer Adriatique ${ }^{10}$, pour ne mentionner que la ville d'Aquilée qui a préservé de nos jours son ancien port fluvial ${ }^{11}$ (fig. 4). D'Aquilée les cargaisons d'amphores étaient dirigées vers les capitales du Norique et de la Pannonie ${ }^{12}$. Les villes de la Gaule Cispadane n'ont livré que de rares exemples de Dressel 20. Concordia Sagittaria, Este et Oderzo (région Vénétie) en ont livré deux chacun. Les amphores bétiques découvertes par Ernesto Canal proviennent de la lagune de Venise ${ }^{13}$. On notera également des trouvailles isolées de Dressel 20 en Gaule Transalpine (fig. 5).

\section{LES IMPORTATIONS DE DRESSEL 20 EN VÉNÉTIE ET DANS LA LAGUNE DE VÉNISE}

La collection d'amphores ayant fait l'objet d'étude est constituée de récipients abrités dans une dizaine de villes de la région de Vénétie et du Frioul. Les enquêtes de recensement

\footnotetext{
${ }^{9}$ Modrzewska-Pianetti 2001: fig. 2.

${ }^{10}$ Toniolo 1989: 56 ; Modrzewska-Pianetti, Pianetti 2003 ; 2012.

${ }^{11}$ Carre, Maselli Scotti 2001.

12 Tassaux 2004: fig. 1-4.

${ }^{13}$ Modrzewska-Pianetti 2000: fig. 59 ; Canal 2013: 441, fig. 165, Dressel 20 issu du canal Catena.
} 


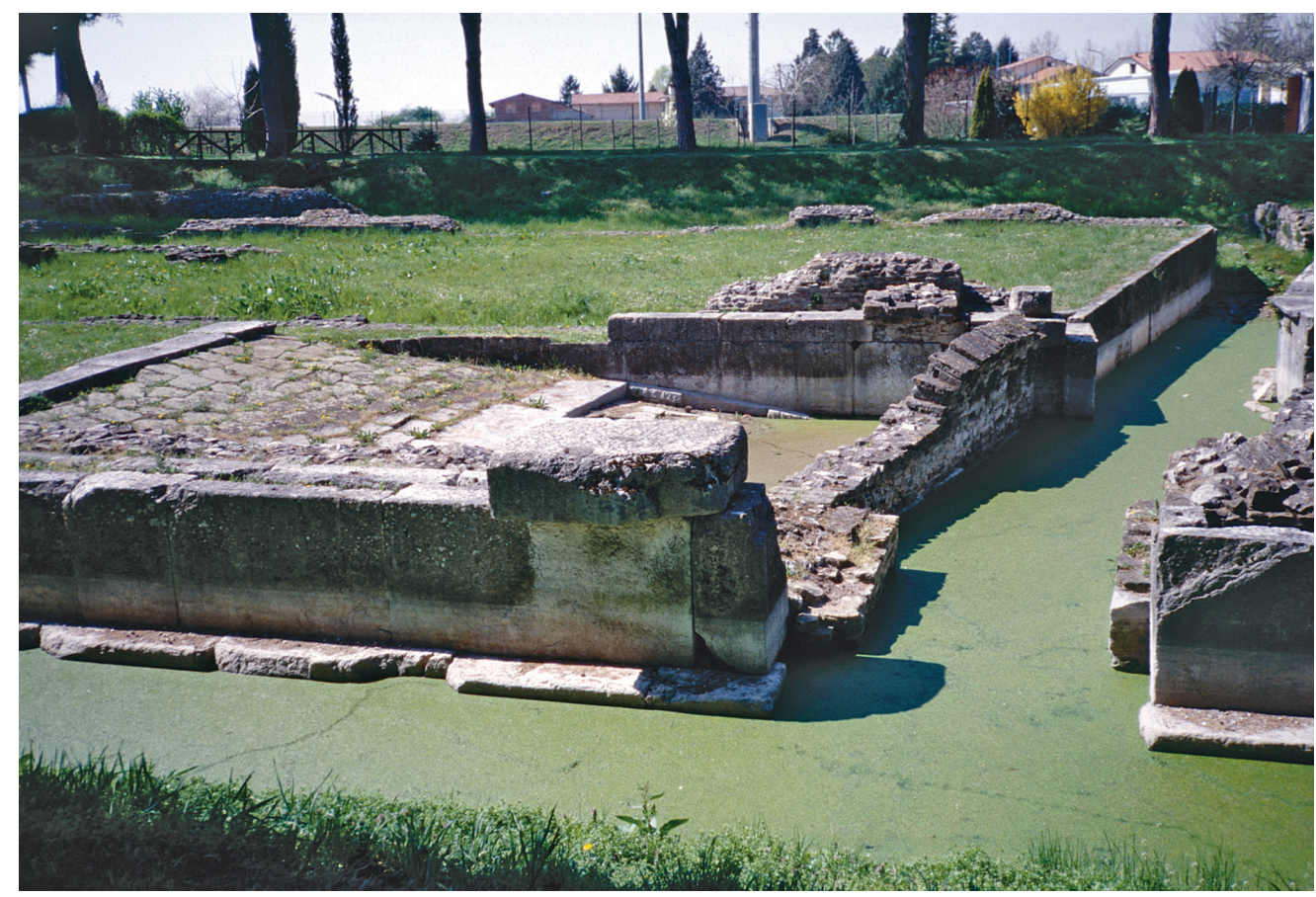

4. Vestiges de l'ancien port romain à l'Aquilée (phot. I. Modrzewska-Pianetti).

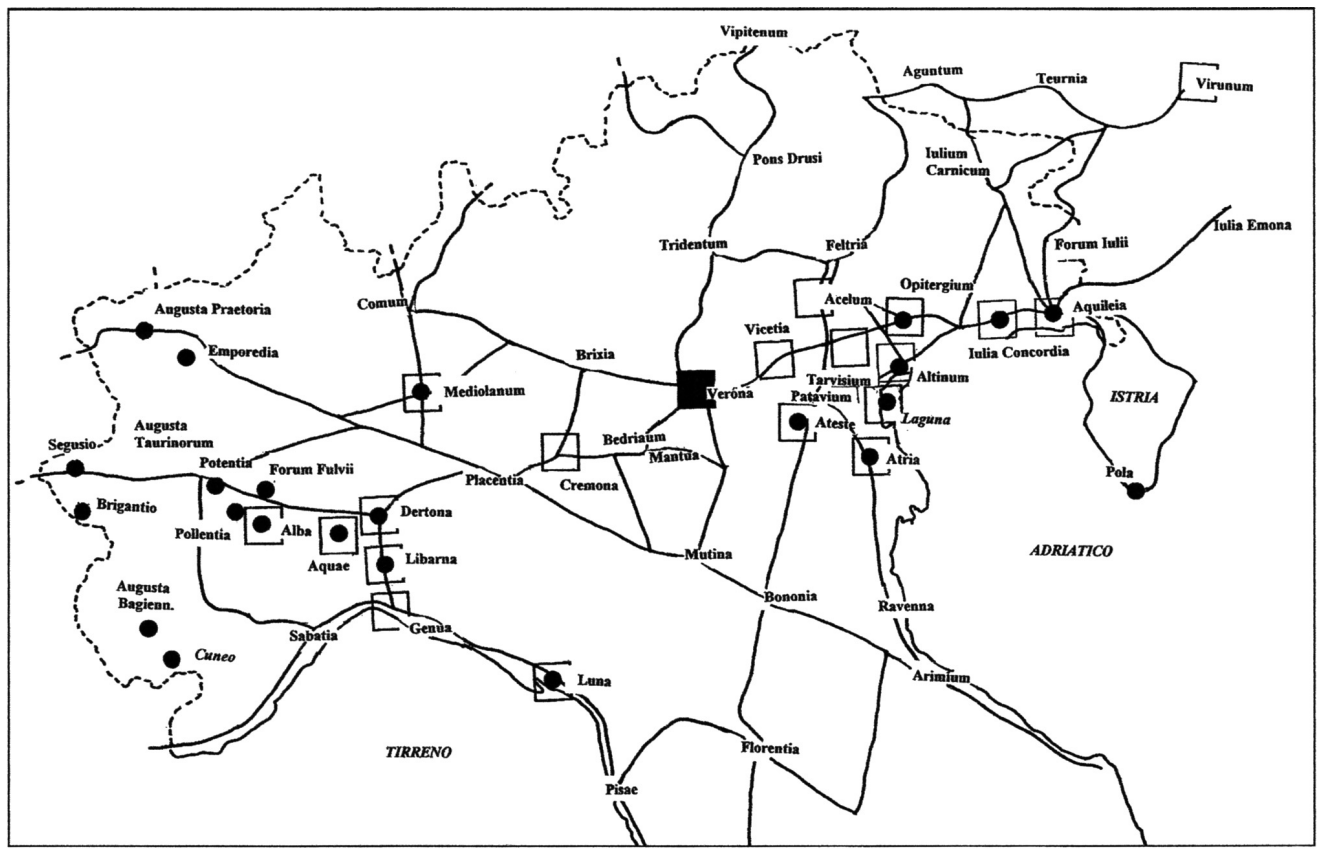

5. Lieux de trouvaille des amphores bétiques en Italie septenterionale (rond - Dressel 20, carré - amphores à garum) (plan : I. Modrzewska-Pianetti). 
réalisées dans les musées ont permis d'établir ce qui suit. Les amphores importées de Bétique se trouvent actuellement dans les dépôts du Musée National d'Aquilée ${ }^{14}$, au Musée de la ville d'Oderzo (2 amphores) ${ }^{15}$, à la bibliothèque municipale de Concordia Sagittaria (2 amphores ; fig. 6) ${ }^{16}$, au Musée National d'Altino (une amphore en dépôt à Ca'delle Anfore ; fig. 7) ${ }^{17}$ et au Musée National d'Este près de Padoue (2 amphores) ${ }^{18}$. Conservés il y a peu de temps encore dans le dépôt privé d'Ernesto Canal au Cannaregio à Venise, trois fragments d'amphores provenant de la lagune de Venise viennent d'être transférés dans la réserve du Musée Archéologique de Venise ${ }^{19}$. Il n'y a en revanche point de Dressel 20 dans les réserves de musées et dans les collections de Trévise, Vicence, San Donà di Piave (Musée de l'Assainissement qui abrite entre autres des trouvailles de l'antique Heraclia), Feltre, Asolo, Vérone en région préalpine et Rovigo, dans la plaine du Pô. Le lot d'amphores utilisées pour le drainage d'un terrain marécageux (première moitié du Ir siècle ap. J.-C.) mis au jour dans le sud de Padoue contient une anse Dressel 20 portant le timbre OM [... et une amphore Dressel 7-11 ${ }^{20}$. En Polésine, les sites de la villa romaine de Corte Cavanella sur l'Adige, d'Adria et de la villa romaine de San Basilio sur le Pô ont livré quelques amphores isolées à huile d'olive bétique ${ }^{21}$. Le matériel amphorique dégagé dans le delta du Pô, toujours en Polésine, compte, outre les amphores bétiques à huile, des amphores à conserves de poisson, un peu plus nombreuses que les premières. Les amphores de type Dressel 20 manquent dans les registres de la réserve du laboratoire du Piovego de l'Université de Padoue ; celle-ci abrite néanmoins quelques amphores Dressel 38 et Beltran II B ${ }^{22}$. Un lot relativement important de 27 amphores Dressel 7/8 d'importation faisant partie d'une trouvaille faite à Vérone, sur la rive droite de l'Adige, se trouve actuellement en dépôt dans les collections de la Tombe de Juliette ${ }^{23}$. Deux récipients ont été prêtés aux UTE - Services Techniques de la Ville de Vérone ${ }^{24}$. Les amphores bétiques abritées au dépôt de la Tombe de Juliette représentent 4\% du mobilier amphorique (divers types) issu d'un magasin portuaire mis au jour sur les bords de l'Adige. À Oderzo, les amphores bétiques constituent 1\% du matériel amphorique découvert à ce jour, tout comme à Altino ${ }^{25}$. À Padoue, les amphores bétiques représentent plus de $3 \%$ des trouvailles ${ }^{26}$.

\footnotetext{
${ }^{14}$ Cf. commentaire ci-après, p. 399-401.

${ }^{15}$ Mazzocchin 2011: fig. 6 ne mentionne que 4 amphores à conserves de poisson.

${ }^{16}$ Modrzewska 1995a: pl. XB, XC.

${ }^{17}$ Modrzewska 1995a: pl. XA ; Mazzocchin 2011: fig. 5 ne fait pas mention de cette amphore.

${ }_{18}$ Toniolo 1989: 55, fig. 44 ne mentionne qu'une de ces amphores, bien que l'autre soit également exposée.

${ }^{19}$ Modrzewska 1995a: pl. XE a, b ; Canal 2013: fig. 165.

${ }^{20}$ Mazzocchin, Tuzzato (éd.) 2007: 130, fig. 8.

${ }^{21}$ Modrzewska 1995a: fig. 13-14.

${ }^{22}$ Modrzewska 1995a: fig. IIB a-c, IIC a-c.

${ }^{23}$ Observation de l'auteur sur place.

${ }^{24}$ Modrzewska 1995a: pl. ID, IE.

${ }^{25}$ Observation de l'auteur sur place.

${ }^{26}$ Mazzocchin 2011: fig. 7-8.
} 
6. Une des deux amphores Dressel 20 trouvées à Concordia Sagittaria, Vénétie (phot. I. Modrzewska-Pianetti).

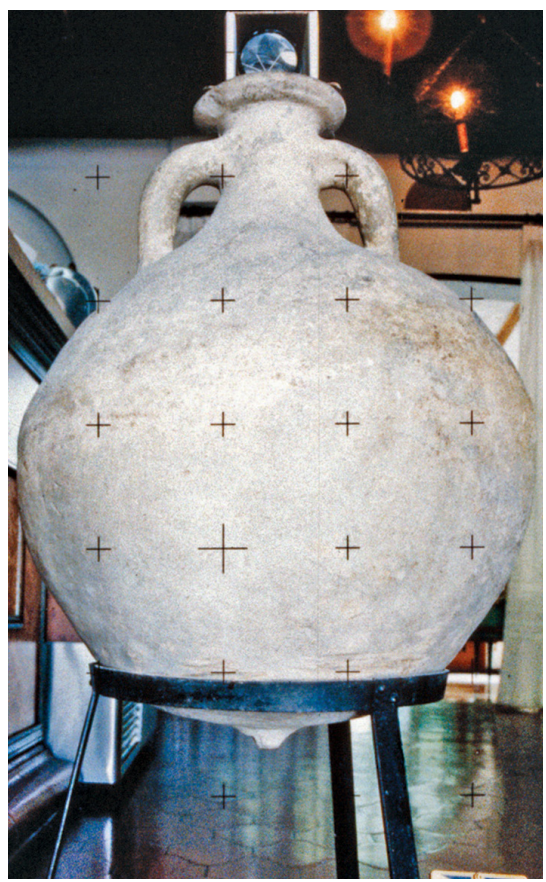

7. Amphore trouvée dans un champ aux environs d'Altino, en dépôt à Cà delle Anfore à Altino (photogrammetrie S. Magro, Padoue ; archives de l'auteur).
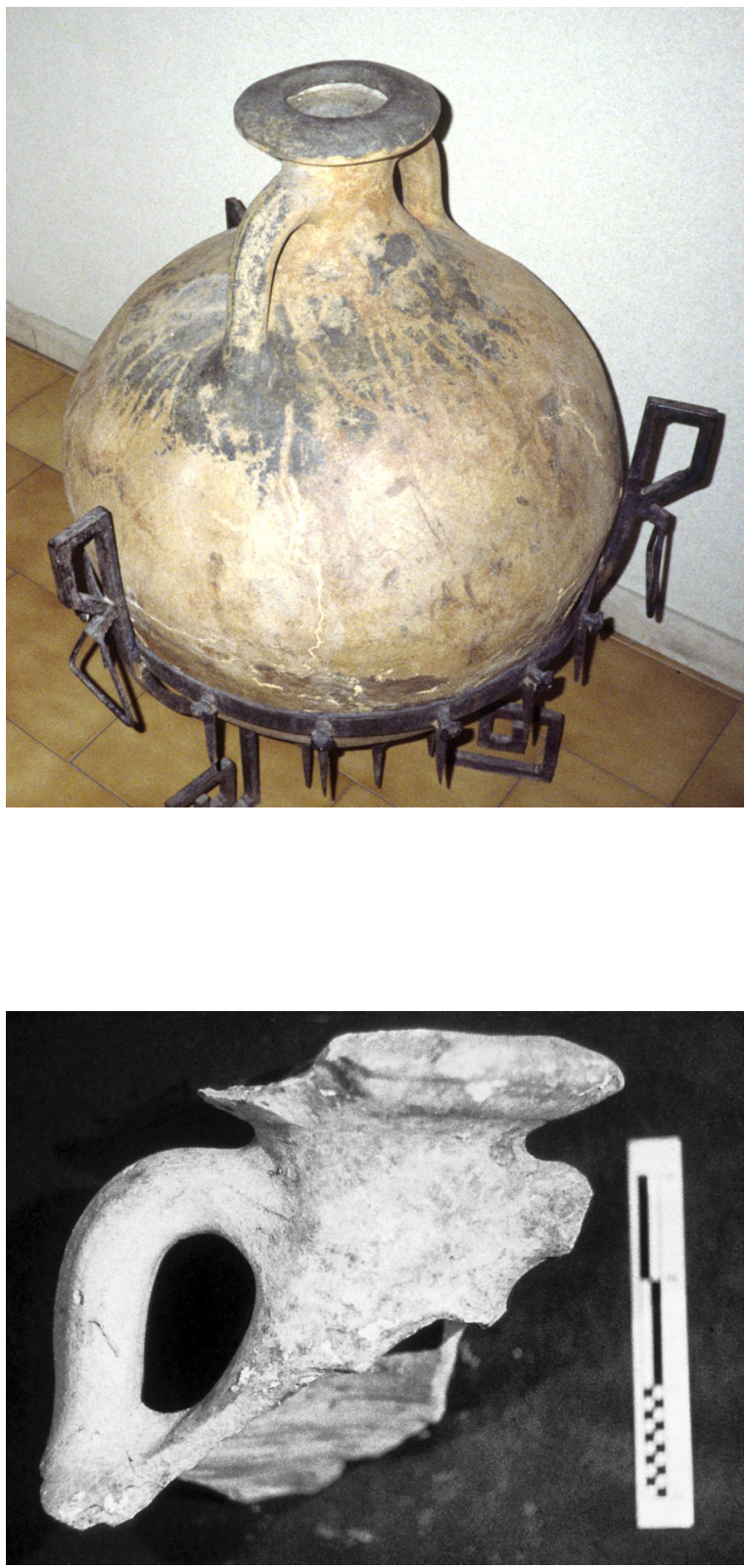

8. Fragment d'amphore Dressel 20 découvert par Ernesto Canal dans le canal Catena, lagune de Venise (phot. I. Modrzewska-Pianetti). 
Dans la lagune de Vénise, les quelques centaines de trouvailles - qui sont le fruit de près de 50 années de travaux de prospection réalisés par Ernesto Canal, inspecteur honoraire de la lagune - ont également livré des amphores bétiques, mais celles-ci ne représentent même pas $1 \%$ de tous les effectifs (différents types de conteneurs). Des fragments de Dressel 20 ont été trouvés dans le canal San Felice dans le nord de la lagune ; le canal Rigà a livré une lèvre et un col fragmentaire Dressel 20 ainsi que la partie supérieure d'un Dressel 8, le canal Catena - un Dressel $38^{27}$. Des fragments de Dressel 20 font partie du mobilier amphorique, dégagé aux environs de l'île d'Ottagono, à l'ouest de Venise, à côté de l'île de Burano et dans le canal Catena, à l'est de Venise (fig. 8) ${ }^{28}$. Dans les trouvailles lagunaires prédominent les amphores adriatiques de types Lamboglia 2, Dressel 6 A et B, Forlimpopoli, les amphores nord-africaines et les amphores LR 2 tardives ${ }^{29}$. À l'époque tardo-antique on observe la prédominance des importations amphoriques d'Afrique du Nord et de l'est méditerranéen. Quant au dénombrement typologique des amphores d'importation, les proportions varient, comme l'ont très bien démontré les études faites à Torcello et sur l'île de San Lorenzo di Ammiana. Les amphores romaines et les amphores tardo-antiques ont été réutilisées dans différents contextes : construction d'ouvrages de défense en fascine, recouvrement de fours de verriers (le cas de Torcello) ou inhumations d'enfants (inhumations en amphores découvertes sur l'île de San Lorenzo di Ammiana) ${ }^{30}$.

À l'époque tardo-antique appartient le fragment d'amphore lusitanienne Almagro 50 mis au jour non loin de l'embouchure de l'Adige, sur le site de la villa romaine de Corte Cavanella (province Rovigo), utilisée jusqu'au début du V $V^{\mathrm{e}}$ siècle ap. J.-C. D’autres amphores originaires de la péninsule Ibérique, à savoir les Dressel 7-11 et Dressel 20, y étaient parvenues avant, ce qu'il faut noter, car il s'agissait non pas d'une ville mais d'une simple mansio, sur la voie de navigation interlagunaire, où subsistent encore de nos jours les vestiges d'un port et de bateaux ${ }^{31}$.

\section{LES TROUVAILLES D’AMPHORES À ESTE, PRÈS DE PADOUE (RÉGION DU VÉNÉTIE)}

La situation géographique d'Este, ville établie sur l'Adige, sur la voie romaine Aemilia Minor qui reliait Bologne à Padoue et qui rejoignait la via Postumia, a toujours favorisé les importations de denrées alimentaires ${ }^{32}$. Dès l'entre-deux-guerres plusieurs découvertes de dépôts d'amphores ont été faites dans la ville d'Este ${ }^{33}$. Sur 54 amphores abritées au Musée National d'Este, seules 12 sont de provenance incertaine. Parmi ces 54 récipients se trouvent 3 amphores bétiques à conserves de poisson de type Dressel 38, Dressel 8 similis

\footnotetext{
${ }^{27}$ Modrzewska-Pianetti 2000: fig. 56-59.

${ }^{28}$ Modrzewska-Pianetti 2000: fig. 59.

${ }^{29}$ Modrzewska-Pianetti 2000: 85-89 ; Gelichi, Moine (éd.) 2012: 29-33.

${ }^{30}$ Leciejewicz 2000 ; Canal 2013: 391 ; Modrzewska-Pianetti 2016.

${ }^{31}$ Scarfi, De Min, Peretto (éd.) 1986: 237-257.

32 Bosio 1970: 115-120.

${ }^{33}$ Toniolo 1989: 45-47.
} 
et Dressel 9. Dans les 49 qui restent on note 26 amphores adriatiques Dressel 6 A et 6 B et 12 amphores apuliennes ${ }^{34}$. Dans l'ensemble du matériel amphorique provenant d'Este, les amphores à huile et à conserves de poisson de Bétique représentent moins d'un pour cent $(0,9 \%)$. Les amphores adriatiques Dressel 6 A et B constituent $48 \%$ du mobilier amphorique mis au jour par les fouilles plus anciennes. Les trouvailles plus récentes d'amphores réutilisées pour le drainage au moment de l'implantation de la nécropole romaine ne contiennent point d'amphores Dressel 20, le site n'ayant fourni que 4 amphores Dressel 7-11 ${ }^{35}$. Dans les trouvailles plus anciennes, les amphores apuliennes représentent $22 \%$ de l'ensemble du matériel amphorique (d'après le catalogue). L'étude des relations quantitatives au sein de tous ces effectifs montre bien que les importations de Bétique représentent une part mineure par rapport à celles d'Apulie ou de la côte adriatique - ce qui ne fait pas exception à la règle dans la structure des dépôts d'amphores dans d'autres villes de la Cisalpine, où les importations bétiques appartiennent visiblement à la catégorie de produits de luxe. D'après les calculs de F. Tassaux, à Trente il y a 2 fragments de Dressel 20 et 17 amphores Dressel 7-11, à Este - 3 amphores Dressel 7-11 sur 100 autres types. De la période augustéenne pour la première décennie du $\mathrm{I}^{\mathrm{er}}$ siècle ap. J.-C. à Vérone, on note environ 27 amphores et fragments d'amphores Dressel 7-11 sur $250{ }^{36}$. À titre de comparaison, l'auteur rapporte les données concernant les sites transalpins : ainsi, 37 amphores Dressel 7-11 à Magdalensberg, 4 à Emona et une à Poetovio ; mais déjà pour l'époque julioclaudienne on note 11 amphores Dressel 7-11 à Poetovio, 12 à Carnuntum, 5 à Vindobona et 2 à Vetus Salina ${ }^{37}$. Au total, on compte environ 50 importations d'amphores bétiques en deux siècles sur - pour la même période - 59 importations d'amphores rhodiennes, cnidiennes et celles de $\operatorname{Kos}^{38}$. En Rhétie, à Coire, furent découverts quelques fragments d'amphores bétiques à garum, tandis que les Dressel 20 représentent $27 \%$ de l'ensemble des importations amphoriques parvenues dans cette région du $\mathrm{I}^{\mathrm{er}}$ jusqu'au début du $\mathrm{III}^{\mathrm{e}}$ siècle ap. J.-C. ${ }^{39}$. La plupart des amphores mises au jour à Este appartiennent à la période du $\mathrm{I}^{\mathrm{er}}$ siècle av. J.-C. au I ${ }^{\mathrm{er}}$ siècle ap. J.-C. ${ }^{40}$. Les trouvailles de récipients plus tardifs nordafricains et orientaux sont peu nombreuses ${ }^{41}$. Les amphores apuliennes apparaissent en Gaule Cisalpine dans le troisième quart du $\mathrm{I}^{\mathrm{er}}$ siècle av. J.-C., avant l'arrivée de récipents adriatiques Dressel 6 A et B. Dans le débat sur l'usage des amphores apuliennes, il semble juste de soutenir l'opinion de Nino Lamboglia selon laquelle ces récipients étaient destinés au conditionnement de l'huile d'olive fabriquée dans la région de Brindisi ${ }^{42}$. Connues aussi de Vérone, de Padoue et de Polésine dans le delta du Pô, les amphores apuliennes restent un témoignage important des échanges commerciaux entre l'Apulie et les régions

\footnotetext{
34 Toniolo 1989: 47-55, catalogue.

${ }^{35}$ Michelini, Mazzocchin 1998: fig. 9.

${ }^{36}$ Tassaux 2004: n. 82.

37 Tassaux 2004: 179.

${ }^{38}$ Bezeczky 1994: 121-122.

39 Tassaux 2004: 159 et n. 122.

40 Toniolo 1989: 55.

${ }^{41}$ Toniolo 1989: fig. 45-47.

${ }^{42}$ Lamboglia 1955: 262-263; Pesavento Mattioli (éd.) 1992: 44, discussion.
} 
de Vénétie et du Frioul au ${ }^{\text {er }}$ siècle av. J.-C. ${ }^{43}$. Les amphores parvenaient en Vénétie via le port d'Adria, du moins jusqu'à la modification des conditions naturelles, et via le port d'Altinum ${ }^{44}$. Outre cela, grâce aux travaux d'Ernesto Canal dans la lagune de Venise, il est possible d'indiquer la direction du trafic commercial via l'île d'Ottagono vers Padoue, par les embouchures des Medoacus Maior et Minor (les Brenta et Bacchiglione actuels) ${ }^{45}$. Une amphore Dressel 20 fut découverte dans les environs de la ville antique d'Altinum, quelques amphores bétiques à conserves de poisson se trouvent dans les collections du Musée National d'Altino. Le petit nombre d'amphores Dressel 20 mises au jour sur le territoire de l'actuelle Vénétie résulte sans doute du fait que l'industrie de l'huile d'olive était aussi implantée dans la péninsule de l'Istrie ${ }^{46}$. Fabriquée en outre dans la région des lacs Côme et Garde, l'huile d'olive y était conditionnée dans des conteneurs spéciaux, désignés dans la littérature du terme « troncoconiche $»{ }^{47}$.

Un nombre légèrement plus haut d'importations d'amphores bétiques à conserves de poisson laisse croire que la demande de denrées de ce type était plus forte de la part des populations de la Cisalpine que celle concernant l'huile d'olive bétique. Vérone fut - comme il a été déjà signalé - un importateur notable de conserves de poisson, mais Aquilée comptait aussi parmi les destinataires d'exception de produits bétiques. En Vénétie, la fabrication de conserves de poisson est attestée uniquement à Corte Cavanella dans la région de Rovigo en Polésine ${ }^{48}$. On note la présence d'à peine 3 amphores à defrutum bétique de type Haltern 70 à Concordia Sagittaria et de 2 autres dans la villa de San Basilio en Polésine ${ }^{49}$. Les amphores vinaires adriatiques de type Dressel 6 A sont très nombreuses dans toutes les trouvailles ${ }^{50}$. Il reste à démontrer l'existence possible de microrégions de commerce, comme c'est le cas d'Oderzo et de Vérone, avec la présence d'amphores à différents usages appelées « collo ad imbuto » (Portorecanati). Les amphores de ce type sont absentes d'Altino, de Padoue et de Vicence. Par ailleurs, la structure typologique du matériel amphorique issu de chacune de ces villes est différente ${ }^{51}$. Les amphores à huile istriennes de type Dressel 6 B servaient indifféremment au conditionnement de conserves de poisson, ce qui rend difficile l'établissement du rapport quantitatif contenant/contenu. Toutefois, il ne fait pas de doute que dans les trouvailles provenant des villes mentionnées de Vénétie et de la lagune de Venise prédominent les amphores nord-italiques. Les amphores de type Dressel 6 A furent aussi fabriquées au Picenum et exportées en masse jusqu'au milieu du ${ }^{\mathrm{er}}$ siècle ap. J.-C. Quant au vin exporté habituellement dans les amphores Dressel 6 A, puis dans les Forlimpopoli et Dressel 2-4 de fabrication istrienne, il ne faut

\footnotetext{
${ }^{43}$ Cipriano, Carre 1989: 68-74.

${ }^{44}$ Rosada 1990: 154-155 ; Tirelli 2001.

${ }^{45}$ Modrzewska-Pianetti, Pianetti 2003 ; Canal 2013: 143-159.

${ }^{46}$ Carre 1985: 218 ; Pesavento Mattioli 1992: 44-45.

${ }^{47}$ Carre 1985: 224-225, 231-232, fig. 6.

48 Toniolo 1989: n. 41.

${ }^{49}$ Modrzewska 1995a: pl. XIVa-c.

${ }^{50}$ Mazzocchin 2011: fig. 5 pour Padoue et Altino, fig. 6 pour Oderzo et Vérone.

${ }^{51}$ Pesavento Mattioli (éd.) 1992: 47; Mazzocchin 2011: fig. 5-6, 8-10.
} 
pas oublier qu'il était aussi conditionné dans des tonneaux, question très peu étudiée jusqu'à présent ${ }^{52}$. F. Tassaux note que dans le matériel amphorique de Magdalenberg appartenant à la période julio-claudienne on compte 45\% des Dressel $6 \mathrm{~B}$ à huile d'olive, 15 Dressel $6 \mathrm{~A}$ à vin adriatique, $15 \%$ des Dressel 4 et $11 \%$ des amphores bétiques à sauces et conserves de poisson. On constate par ailleurs que les deux types d'amphores - à savoir : Dressel 6 A et B - sont majoritaires dans les mobiliers provenant de Virunum. Tout comme à Este, le nombre des amphores bétiques à huile d'olive et à conserves de poisson n'égale même pas $1 \%$ des amphores adriatiques.

\section{LES AMPHORES BÉTIQUES À AQUILÉE (RÉGION DU FRIOUL)}

La ville d'Aquilée a livré de nombreuses amphores. Le dépôt d'amphores du Musée National d'Aquilée présente une structure différente de celle des trouvailles de surface, moins nombreuses. Le dépôt du Musée compte environ 1000 amphores entières et fragmentaires ${ }^{53}$ de différents types, dont Dressel 20, Dressel 14 et Dressel 38/39. Dans le matériel attribué à la période comprise entre le $\mathrm{II}^{\mathrm{e}}$ et le milieu du $\mathrm{I}^{\mathrm{er}}$ siècle av. J.-C. dominent les amphores de type Lamboglia 2 qui sont cinq fois plus nombreuses que les gréco-italiques importées du sud de la péninsule Apennine ${ }^{54}$. À cette époque, Aquilée voit affluer de petites quantités d'huile d'olive conditionnée dans des conteneurs apuliens et des quantités similaires de vin tyrrhénien dans des amphores Dressel 1. Au Ir siècle ap. J.-C., la gamme de produits importés par Aquilée s'élargit. Parmi les nouveautés on compte le vin campanien conditionné dans des amphores Dressel 2-4, mais aussi une plus grande quantité de conserves de poisson de Bétique dans des amphores Dressel 14 et Dressel 38/39 ${ }^{55}$. En dehors d'Aquilée, une amphore Dressel 14 fut découverte à Corte Cavanella sur l'Adige. Il est toutefois difficile de savoir si elle est issue d'un atelier implanté dans la région de Grenade ou de l'un des centres plus importants de production amphorique en Lusitanie ${ }^{56}$. Dans le mobilier amphorique d'Aquilée attribué au Irr siècle ap. J.-C., les amphores à conserves de poisson sont plus nombreuses que les amphores Dressel 20. Pour la même période, on note une faible présence d'amphores vinaires importées de Rhodes. Dans cette ville, le nombre de trouvailles d'amphores Dressel 20 est comparable à celui d'amphores Dressel 25. Les Dressel 25 sont originaires de l'est méditerranéen, ce dont semble témoigner la composition de la pâte céramique, mais il est impossible de définir plus exactement leur lieu de fabrication. Les amphores de ce type sont connues de Vicence et de Padoue ${ }^{57}$. Les sites d'Aquilée ont en outre livré des amphores à fond plat, fabriquées en Émilie-Romagne, dans des ateliers de Forlimpopoli et de Sant'Arcangelo di Romania ${ }^{58}$. Certaines d'entre

\footnotetext{
${ }^{52}$ Tassaux 2004: 177-178, 182-183 ; Desbat 1997 ; Marlière 2001: fig. 101.

${ }^{53}$ Cipriano, Carre: 1987.

${ }^{54}$ Cipriano, Carre 1987: fig. 1.

${ }^{55}$ Cipriano, Carre 1987: fig. 2.

${ }^{56}$ Modrzewska 1995a: pl. XIIIC a ; Bernal Casasola, Navas Rodríguez 1996.

${ }^{57}$ Mazzocchin, Tuzzato (éd.) 2007: 130 ; Mazzocchin 2011: 246-248, fig. 1-3.

${ }^{58}$ Aldini 1978: 242-243 ; Carre 1985: 229.
} 
elles sont timbrées au nom de $Q$. Ninius Secundus, un potier connu de Picenum ${ }^{59}$. Destinées très vraisemblablement au confectionnement du vin, elles ont été fabriquées dès le $\mathrm{I}^{\mathrm{er}}-\mathrm{III}{ }^{\mathrm{e}}$ siècle ap. J.-C. On note leur présence dans les trouvailles de la lagune de Venise ${ }^{60}$. Le matériel amphorique issu des sites lagunaires et des sites terrestres de la région de Vénétie affiche une domination évidente des Dressel $6 \mathrm{~A}$ et B adriatiques ${ }^{61}$. À Aquilée, les amphores adriatiques sont 30 fois plus nombreuses que les Dressel 20 et les amphores rhodiennes. Il en est de même pour le matériel amphorique livré par les fouilles de Teurnia et de Virunum, au Norique, où prédominent, dans les proportions comparables, les importations adriatiques.

$\mathrm{Au} \mathrm{II}^{\mathrm{e}}-\mathrm{III}^{\mathrm{e}}$ siècle, l'Aquilée connaît une diminution notable d'importations ${ }^{62}$ et les directions des échanges commerciaux changent. La ville voit arriver d'Afrique du Nord les conteneurs à huile et à conserves de poisson dits africains ou tripolitains. Mais les quantités d'importations nord-africaines sont proches de celles de Dressel 20 bétiques. Les importations d'amphores à huile bétiques se présentent comme suit :

- $\mathrm{I}^{\mathrm{er}}$ siècle ap. J.-C. - 8 Dressel 20 contre 20 Dressel 14 et Dressel 38/39 ;

- $\mathrm{II}^{\mathrm{e}}-\mathrm{III}^{\mathrm{e}}$ siècle -19 Dressel 20/23, absence d'amphores à conserves de poisson ${ }^{63}$;

- IV - V e siècle - 20 Dressel 23, absence d'amphores à conserves de poisson bétiques.

Les changements dans la structure de la population que connût la Gaule Cisalpine aux $\mathrm{IV}^{\mathrm{e}}-\mathrm{VI}^{\mathrm{e}}$ siècles ne font interrompre les importations de produits alimentaires ni à Aquilée ni à Torcello, ce qui est illustré par le dessin reproduit dans l'ouvrage cité ${ }^{64}$. L'épave d'un bateau du milieu du II $^{\mathrm{e}}$ siècle ap. J.-C., échoué dans la lagune de Grado (arrière-port d'Aquilée) et exploré entre 1987 et 1998, a livré 600 amphores nord-africaines, amphores de tradition de Kos, Pompei 6, Knossos 19, et locales : Dressel 19, Forlimpopoli et Dressel $21 / 22^{65}$. Les analyses de laboratoire ont démontré que toutes ces amphores étaient utilisées et réutilisées dans le conditionnement de salsamenta (différentes variétés de poisson salé) ${ }^{66}$. À Aquilée, à cette époque tardive, les importations d'huile d'olive bétique ne cessent point, mais le produit est désormais conditionné dans des amphores plus petites que les Dressel 20, à savoir les Dressel $23{ }^{67}$. Au IV $-V^{\mathrm{e}}{ }^{\mathrm{e}}$ siècle, les produits africains parvenaient dans des amphores cylindriques et dans des spatheia. Dans le matériel archéologique, la quantité d'amphores tunisiennes égale six fois celle de conteneurs égéens et bétiques - ce qui est tout à fait compréhensible, compte tenu du rôle que jouent les régions nordafricaines dans l'approvisionnement du bassin méditerranéen à partir du IVe-VI ${ }^{\mathrm{e}}$ siècle.

\footnotetext{
${ }^{59}$ Cipriano, Carre 1987: 286.

${ }^{60}$ Modrzewska-Pianetti 2000: 50.

${ }^{61}$ Cipriano, Mazzocchin, Pastore: 1998, fig. 6, 11 ; Cipriano 2009: fig. 4, 10 ; Mazzocchin 2011: 281-282.

${ }^{62}$ Cipriano, Carre 1987: fig. 3.

${ }^{63}$ Cipriano, Carre 1987, interprétation de données à partir des fig. 1-4.

${ }^{64}$ Modrzewska-Pianetti 2001: fig. 55.

${ }^{65}$ Auriemma 2000: 32-33, 37.

${ }^{66}$ Auriemma 2000: 41-47.

${ }^{67}$ Cipriano, Carre 1987: fig. 4.
} 
9. Quantités des amphores de différentes origines issues des prospections réalisées à l'Aquilée - présentation en pourcentage (d'après : Gomezel 1997: diagramme 4 ; élaboration graphique : M. Różycka).

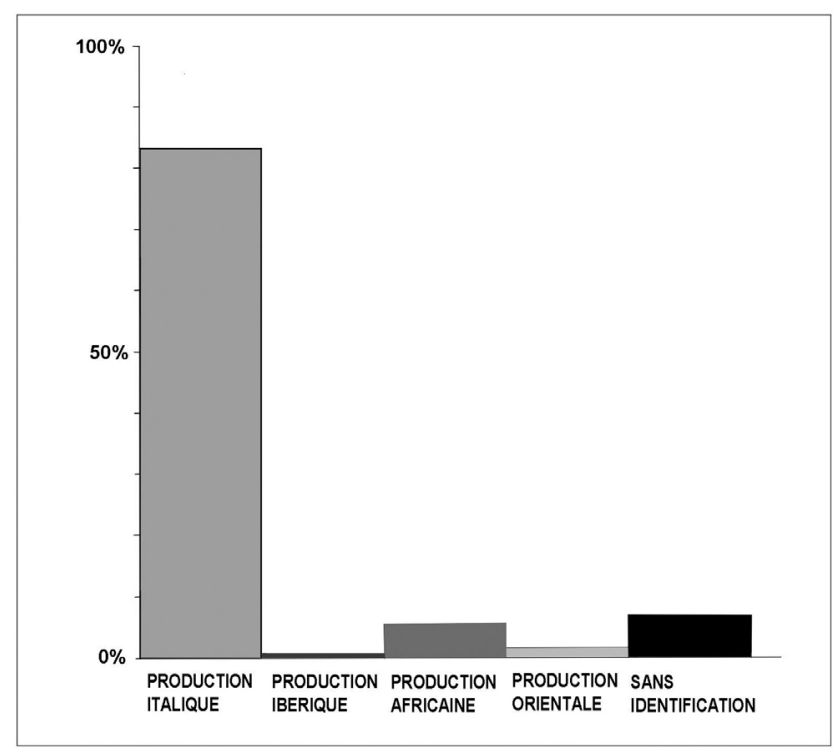

À la même époque, Aquilée voit aussi arriver des amphores égéennes de type Atene M $273{ }^{68}$. La vérité est que, pendant sept siècles, Aquilée est approvisionnée par toutes les régions de la Méditerranée. À partir du Ir siècle ap. J.-C., l'huile d'olive bétique y parvient dans des amphores Dressel 20 et puis, au IV et au début du V $V^{\mathrm{e}}$ siècle, dans des amphores Dressel 23. Au total, parmi les 1000 amphores recensées, on compte environ 50 récipients destinés au conditionnement de l'huile d'olive bétique ${ }^{69}$ - ce qui représente $5 \%$ des importations, alors que dans les autres villes de Vénétie ce chiffre n'atteint même pas un pour cent. Les travaux de prospection réalisés dans les années $90 \mathrm{du}$ siècle dernier ont livré 454 fragments diagnostiques ${ }^{70}$. Dans ce corpus, les rapports quantitatifs entres divers types d'amphores diffèrent de ceux établis pour le matériel amphorique conservé dans le musée d'Aquilée. Les amphores bétiques Dressel 7-11 représentent $0,4 \%$ des trouvailles de surface, ce qui correspond à 2 individus (fig. 9) ${ }^{71}$. À Aquilée, 20 amphores à conserves de poisson constituent $1 \%$ de l'ensemble des trouvailles de surface. Les amphores Dressel 20 représentent $0,2 \%$ des trouvailles de surface, ce qui correspond à un individu. Dans les réserves du Musée, les Dressel 20 représentent $5 \%$ du matériel amphorique, soit 25 individus ${ }^{72}$. Mais, en termes de proportions, dans les deux corpus étudiés (amphores du Musée d'Aquilée et trouvailles de surface provenant de cette ville) la prédominance des amphores adriatiques sur les amphores bétiques est similaire.

\footnotetext{
${ }^{68}$ Cipriano, Carre 1987: 486-487.

${ }^{69}$ D'après les données fournies par Cipriano, Carre 1987: fig. 1-4.

${ }^{70}$ Gomezel 1994: 526.

${ }^{71}$ Calcul effectué à partir des pourcentages : Gomezel 1994: 526.

${ }^{72}$ Gomezel 1994: 526-527 ; Cipriano, Carre 1987: fig. 2-3 ; Tassaux 2004: 184.
} 


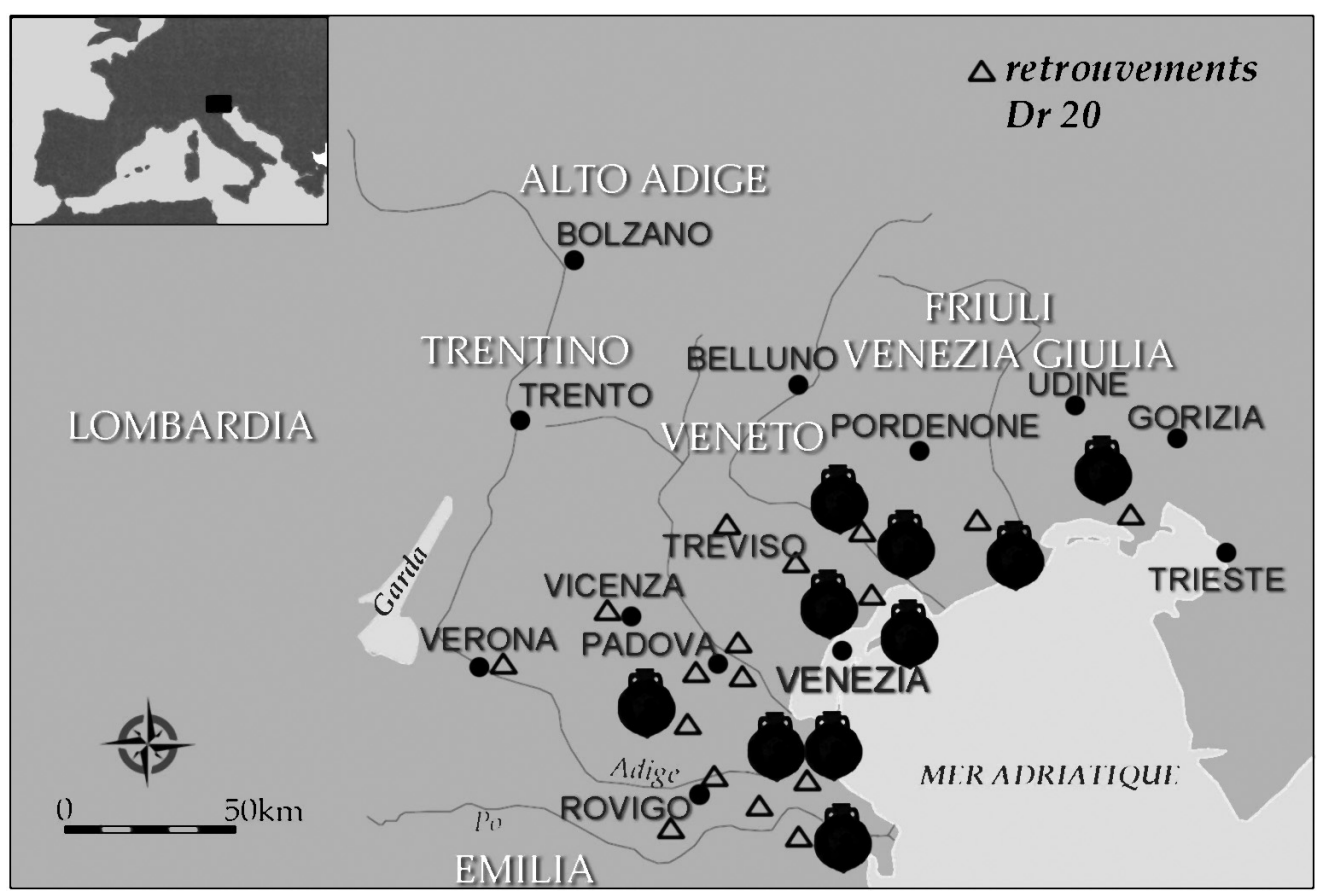

10. Carte de distribution des Dressel 20 en Vénétie et au Frioul (élaboration : I. Modrzewska-Pianetti ; élaboration graphique : M. Różycka).

\section{COMMENTAIRE À LA DISTRIBUTION DES IMPORTATIONS DE BÉTIQUE EN GAULE CISALPINE}

Après l'analyse de toutes ces données, il apparaît que la répartition des trouvailles d'amphores bétiques reste en rapport évident avec le tracé des voies romaines dans cette partie de la Gaule. Sur le territoire de la X Regio Venetia et Histria les trouvailles de Dressel 20 se répartissent le long du littoral de la mer Adriatique (fig. 10). Les données recueilles montrent bien que les importations de l'huile d'olive bétique étaient principalement destinées à la ville d'Aquilée (fig. 11). Il s'agissait d'un produit de luxe qui venait compléter l'offre de l'huile d'olive istrienne, produit de consommation courante. Pour la région côtière de l'Adriatique, la distribution des amphores bétiques à conserves de poisson correspond à celle des Dressel 20. Il en est de même pour la lagune de Venise où, dans les canaux Rigà et Catena, ont été découvertes des amphores Dressel 20 et Dressel $38^{73}$. La répartition des trouvailles fragmentaires de Dressel 20 dans le nord de la lagune indique la direction des importations vers la ville portuaire d'Altinum (l'actuel Altino), sur la côte du Haut Adriatique, d'où - par la via Annia qui rejoignait la via Postumia ou par les fleuves Silis et Plavis - les cargaisons d'huile d'olive et de poisson salé parvenaient à Opitergium (l'actuel Oderzo).

\footnotetext{
${ }^{73}$ Modrzewska-Pianetti 2000: fig. 56.
} 


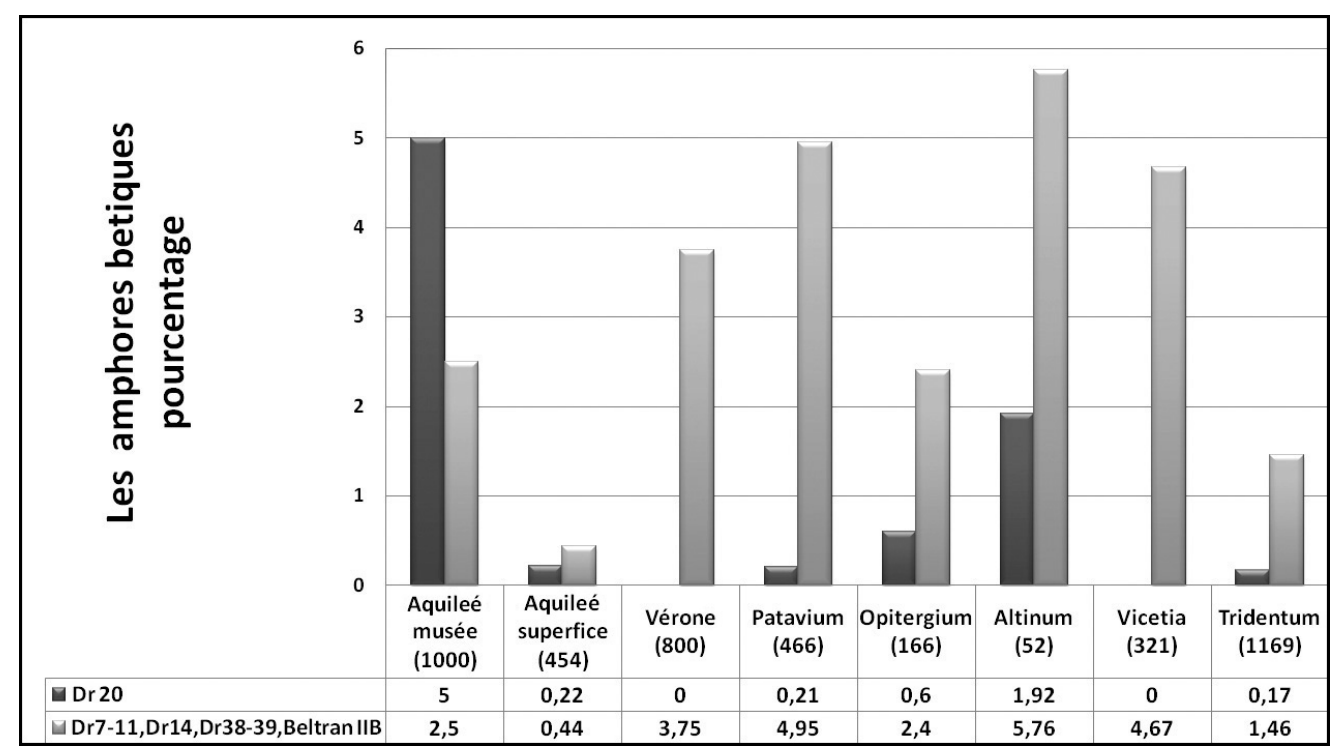

11. Les amphores bétiques Dressel 20, Dressel 7-11, 14, Dressel 38-39, Beltran II B - présentation en pourcentage : Aquilée - amphores du musée, d'après Cipriano, Carre 1987; Aquilée - amphores issues des prospections, d'après Gomezel 1994 ; Vérone, d'après Mazzocchin 2011, données complétées par I. Modrzewska-Pianetti ; Padoue, d'après Mazzocchin 2011, données complétées par I. Modrzewska-Pianetti, d'après Mazzocchin, Tuzzato (éd.) 2007 ; Oderzo, d'après Mazzocchin 2011, données complétées par I. Modrzewska-Pianetti (1 amphore Dressel 20) ; Altino, d'après Mazzocchin 2011, données complétées par I. Modrzewska-Pianetti (3 amphores Dressel 7-11) ; Vicence, d'après Mazzocchin 2011 avec complément de Modrzewska-Pianetti - amphores à conserves de poisson ; Trente, d'après Tassaux 2004 (élaboration : I. Modrzewska-Pianetti ; élaboration graphique : M. Różycka).

L'arrière-pays de la X Regio Venetia et Histria présente une toute autre image des importations. En effet, à Trévise, Vicence et Vérone furent mises au jour les amphores à conserves de poisson provenant de Bétique, mais on n'y a point découvert de Dressel 20. Une seule amphore de ce type est connue de Padoue, où elle fut réutilisée dans le drainage d'un terrain marécageux dans le nord de la ville ${ }^{74}$. Une trouvaille exceptionnelle faite sur la rive de l'Adige, à proximité de l'actuelle Tombe de Juliette à Vérone, celle notamment d'un dépôt d'amphores contenant de nombreux Dressel 7/8 datés de la période augustéenne et Dressel 7-11 de la période claudienne, n'a livré aucune amphore de type Dressel $20^{75}$.

L'examen des caractéristiques morphologiques des amphores Dressel 20 découvertes en Vénétie autorise à penser que la plupart d'entre elles sont parvenues en Gaule Cisalpine sous Claude et Néron, puis sous Trajan. Les importations de formes anciennes de Dressel 20 ne sont point notées avant la fin du Ir siècle av. J.-C. Elles sont absentes des collections du musée d'Aquilée. De très rares Dressel 23 sont connus de la lagune de Venise et d'Aquilée ${ }^{76}$.

\footnotetext{
${ }^{74}$ Mazzocchin, Tuzzato (éd.) 2007: 130.

${ }^{75}$ Mazzocchin 2011: fig. 6 et observations personnelles.

${ }^{76}$ Cipriano, Carre 1987: fig. 4.
} 
Il est donc légitime d'affirmer que les importations de Dressel 20 en Vénétie présentent des quantités infimes par rapport à celles des amphores bétiques à sauces et à conserves de poisson.

La ville d'Aquilée fait néanmoins exception, car on y trouve un nombre plus important de Dressel 20, avec tout de même la prédominance des amphores Dressel 6 A et B. Cette ville témoigne d'ailleurs le mieux de l'ouverture des cités côtières du Haut Adriatique au commerce du vin et de l'huile d'origine adriatique et méditerranéenne orientale, compte tenu du nombre très limité de produits qui viennent de la côte tyrrhérienne de l'Italie. Disposant aussi de l'offre la plus variée de produits d'importation, l'Aquilée est sans aucun doute le plus gros importateur régional de produits bétiques destinés à la population aisée de la ville. En effet, la plupart des habitants se contentaient de l'huile d'olive istrienne et du vin de la plaine du Pô. Dans les collections de la région de Vénétie il n'existe point d'amphores originaires de Gaule Narbonnaise ni d'Hispanie Tarraconaise - ce qui signifie que les marchands et les négociants narbonnais et tarraconais n'ont pas vraiement visé le marché adriatique. L'étude des amphores importées de Bétique réalisée en confrontation avec les résultats des recherches concernant les conteneurs de fabrication locale a bien démontré l'existence des microrégions pour les produits d'importation dans certaines villes romaines en Gaule Cisalpine.

\section{Remerciments}

L'article fut élaboré dans le cadre du projet n ${ }^{\circ}$ UMO-2015/18/M/HS3/00248, Harmonia 7, financé par le Centre National de Recherche Scientifique en Pologne.

Les remercients particuliers de l'auteur pour autorisation d'utiliser les photos provenant d'Archives du Centro para el Estudio de la Interdependencia Provincial en la Antigüedad Clasica (CEIPAC) vont au professeur José Remesal Rodríguez de Universidad de Barcelona.

(traduction : K. Bartkiewicz)

\section{Références}

Aldini, T. 1978: Anfore foropopilensi, ArchClass XXX, 236-245

Auriemma, R. 2000: Le anfore del relitto Grado e il loro contenuto, MEFRA 112/1, 27-51

Bernal Casasola, D., Navas Rodríquez, J. 1996: Los Matagallares. Un centro alfarero romano en Salobreña, Revista de Arqueología 182, 42-51

Bezeczky, T. 1994: Aegean amphorae in Pannonia, Folia Archaeologica 43, 115-125

Blázquez Martínez, J.M., Remesal Rodríguez, J., Rodríquez Almeida, E. 1994: Excavaciones arquéologicas en el Monte Testaccio (Roma). Memoria Campaña 1989, Madrid

Bosio, L. 1970: Itinerari e strade della Venetia romana, Padova

Brecciaroli Taborelli, L. 1987: Per una ricerca sul commercio nella Transpadana occidentale in epoca romana: ricognizione selle anfore di Vercellae, [dans :] Roda, S. (éd.), Atti del Convegno di Studi nel centenario della morte di Luigi Bruzza 1883-1983: Vercelli 6-7 ottobre1984, Vercelli, 129-208 
Canal, E. 2013: Archeologia della laguna di Venezia 1960-2010, Verona

Carre, M.B. 1985: Les amphores de la Cisalpine et de l'Adriatique au début de l'Empire, MEFRA 97/1, 207-245

Carre, M.B., Maselli Scotti, F. 2001: Il porto di Aquileia: dati antichi e ritrovamenti recenti, AAAdr XLVI, 211-243

Carre, M.-B., Matijaši, R., Pesavento Mattioli, S. 1993: Économie, communications / / Economia, comunicazioni, MEFRA 105/1, 373-395

Cipriano, S. 2009: Le anfore olearie Dressel 6B, [dans :] Pesavento Mattioli, S., Carre, M.-B. (éd.), Olio e pesce in epoca romana. Produzione e commercio nelle regioni dell'Alto Adriatico, Atti del Convegno (Padova 16 febbraio 2007), Antenor Quaderni 15, Padova, 175-189

Cipriano, M.T., Carre, M.B. 1987: Note sulle anfore conservate nel Museo d'Aquileia, [dans :] Vita sociale, artistica e commerciale di Aquileia romana, AAAdr XXIX, 479-494

Cipriano, M.T., Carre, M.-B. 1989: Production et typologie des amphores sur la côte adriatique de l'Italie, [dans :] Zevi, F. (éd.), Amphores romaines et histoire économique : dix ans de recherche. Actes du colloque de Sienne 22-24 mai 1986, CEFR 114, Rome, 67-104

Cipriano, S., Mazzocchin, S., Pastore, P. 1998: Padova. Tre casi in aree a diversa funzionalità, [dans :] Pesavento Mattioli, S. (éd.), Bonifiche e drenaggi con anfore in epoca romana : aspetti tecnici e topografici. Atti del seminario di studi Padova 19-20 ottobre 1995, Materiali d'archeologia 3, Modena, 161-174

Desbat, A. 1997: Le tonneau antique. Question techniques et problème d'origine, [dans :] Garcia, D., Meeks, D. (éd.), Techniques et économie antiques et médiévales. Le temps de l'innovation. Actes du colloque d'Aix-en-Provence mai 1996, Travaux du Centre Camille Julian CNRS 21, Paris, 113-120

Gelichi, S., Moine, C. (éd.) 2012: Isole fortunate? La storia della laguna nord di Venezia attraverso lo scavo di San Lorenzo di Ammiana, Archeologia Medievale. Cultura materiale. Insediamenti. Territorio XXXIX, 9-56

Gomezel, C. 1994: Nuovi bolli su anfora dal territorio aquileiese, [dans :] Panciera, S., Nicolet, C. (éd.), Epigrafia della produzione e della distribuzione. Actes de la VII ${ }^{\mathrm{e}}$ rencontre franco-italienne sur l'épigraphie du monde romain (Rome 5-6 juin 1992), CEFR 193, Rome, 525-541

Lamboglia, N. 1955: Sulla cronologia delle anfore romane di età repubblicana (II-I sec. a.C.), Rivista degli Studi Liguri XXI/1, 241-270

Leciejewicz, L. 2000: Torcello antica e medievale alla luce delle nuove ricerche archeologiche, [dans :] Leciejewicz, L. (éd.), Torcello. Nuove ricerche archeologiche, RivArch Supplemento 23, Roma, 87-98

Marlière, E. 2001: Le tonnau en Gaule romaine, Gallia 58, 181-201

Mazzocchin, S. 2011: Trafici comerciali a Vicenza in epoca romana: i dati delle anfore, thèse de doctorat, l'Université de Padoue, Padoue, http://paduaresearch.cab.unipd. it/3469/ (accedé le 10 septembre 2015) 
Mazzocchin, S., Tuzzato, S. (éd.) 2007: Padova, via Aquette 9: nuovi dati dal settore meridionale della città romana, Quaderni di Archeologia del Veneto XXIII, 123-139

Michelini, P., Mazzocchin, S. 1998: Este: la temporanea bonifica od uso funerario di un spazio lungo il fiume, [dans :] Pesavento Mattioli, S. (éd.), Bonifiche e drenaggi con anfore in epoca romana: aspetti tecnici e topografici. Atti del seminario di studi di Padova 19-20 ottobre 1995, Materiali d'archeologia 3, Modena, 223-235

Modrzewska, I. 1995a: Anfore spagnole nel Veneto. Testimionianze dei contatti commerciali Betica-Venetia, Pisa

Modrzewska, I. 1995b: Anfore spagnole nella laguna e terraferma Veneta (Italia), [dans :] Vendre-Saz, M., Pradell, T., Molera, J., Garcia, M. (éd.), Estidis sobre ceramica antiga: Actes del simposi sobre ceramica antica (Barcelona 1993), Barcelona, $123-125$

Modrzewska-Pianetti, I. 2000: Sulla storia della laguna di Venezia nell'Antichità, Światowit Supplement Series A, Antiquity IV, Warszawa

Modrzewska-Pianetti, I. 2001: Importy amfor do Galii Cisalpińskiej: dane archeologiczne z obszarów Cisalpiny, Światowit 3 (44)/A, 143-156

Modrzewska-Pianetti, I. 2016: Amphores romaines tardives découvertes sur l'île de San Lorenzo (lagune de Venise). Discussion et nouveaux constats, EtudTrav XXIX, 135-156

Modrzewska-Pianetti, I., Pianetti, F. 2003: Alla ricerca del porto di Patavium, Saguntum 35, 197-215

Modrzewska-Pianetti, I., Pianetti, F. 2012: La laguna di Venezia in epoca romana, [dans :] Cavalieri, M. (éd.), Industria Apium. L'archéologie : une démarche singulière, des pratiques multiples. Hommage à Raymont Brulet, Louvin, 205-216

Pesavento Mattioli, S. (éd.) 1992: Anfore romane a Padova : ritrovamenti dalla città, Materiali di Archeologia 1, Modena

Remesal Rodríguez, J. 1986: La annona militaris y la exportación del aceite bético a Germania, Madrid

Remesal Rodríguez, J. 2002: Baetica and Germania. Notes on the concept of 'provincial interdependence' in the Roman Empire, [dans :] Erdkamp, P. (éd.), The Roman Army and the Economy, Amsterdam, 293-308

Rosada, G. 1990: La direttrice endolagunare e per acque interne nella decima regio maritima. Tra risorsa naturale e organizzazione antropica, [dans :] Pavan, M., Rosada, G. (éd), La Venetia nell'area padano-danubiana. Le vie di comunicazione. Convegno Internazionale, Venezia 6-10 aprile 1988, Padova, 153-182

Scarfî, M.B., De Min, M., Peretto, R. (éd.) 1986: L'antico Polesine. Testimonianze archeologiche e paleoambientali. Catalogo delle esposizioni di Adria e di Rovigo febbraio-novembre 1986, Padova

Tassaux, F. 2004: Les importations de l'Adriatique et de l'Italie du Nord vers les provinces danubiennes de César aux Sévères, [dans :] Urso, G. (éd.), Dall'Adriatico al Danubio. L'Illirico nell'eta greca e romana. Atti del convegno internazionale, Cividale del Friuli, 25-27 settembre 2003, Pisa, 167-205 
Tirelli, M. 2001: Il porto di Altinum, [dans :] Zaccaria, C. (éd.), Strutture portuali e rotte marittime nell'Adriatico di età romana. Atti della XXIX settimana di studi aquiliesi 23-23 maggio 1998, AAAdr 46, CEFR 280, Roma, 295-316

Toniolo, A. 1989: Anfore conservate nel magazzino del Museo di Este, [dans :] Calzolari, M. (éd.), Civiltà padana, Archeologia e storia del territorio I/1988, Modena, 45-74 


\section{ÉTUDES et TRAVAUX XXX / 2017}

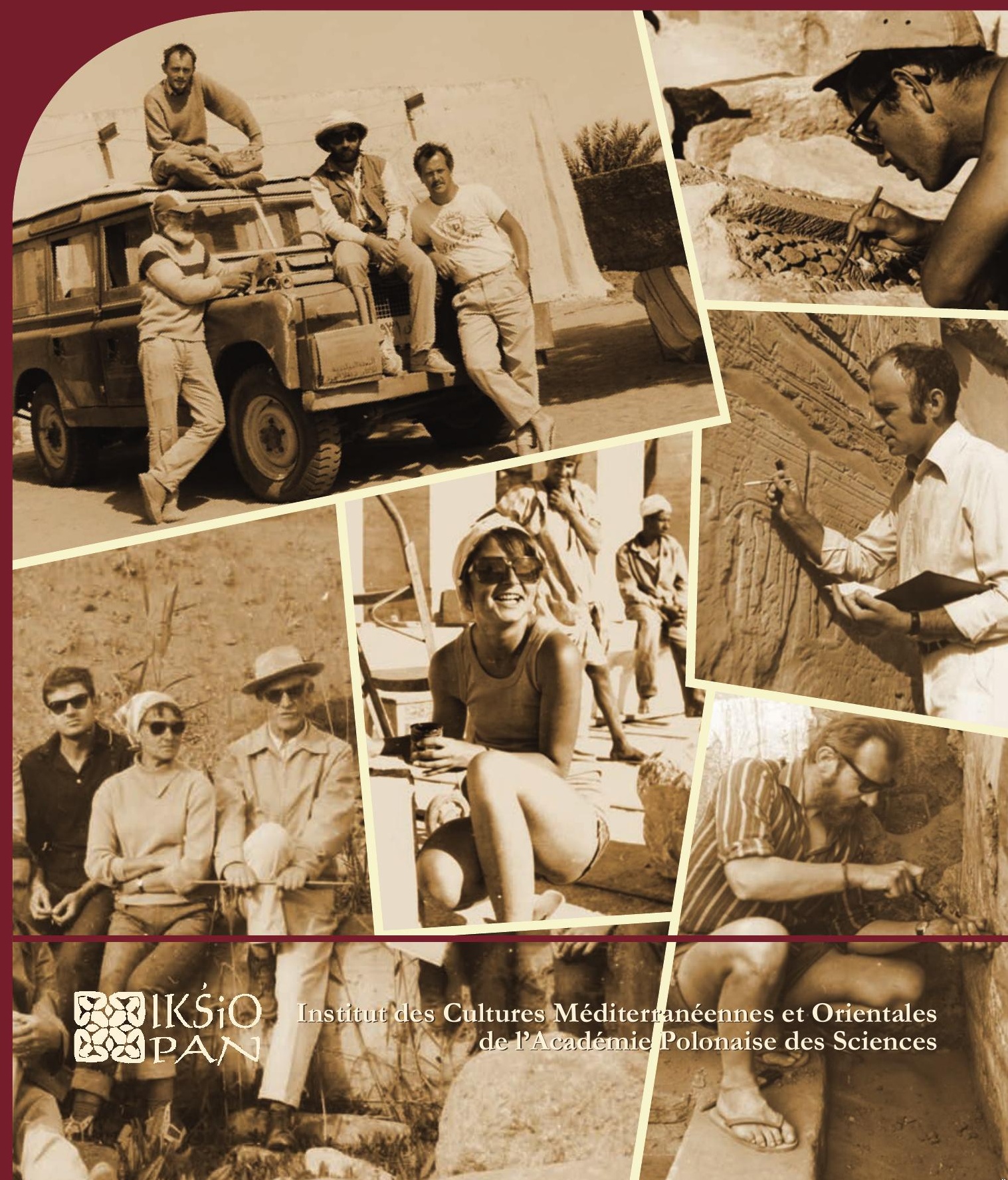




\title{
COMITÉ DE RÉDACTION SCIENTIFIQUE
}

Maciej Makowski - rédacteur en chef

Jadwiga Iwaszczuk - rédacteur et sécretaire de la rédaction

Mariusz Drzewiecki - rédacteur

Maciej G. Witkowski - rédacteur

\section{CONSEIL SCIENTIFIQUE DU JOURNAL}

M. Kobusiewicz (IAE PAS, Warszawa), E. Laskowska-Kusztal (IMOC PAS, Warszawa),

D. Michaelides (University of Cyprus, Nicosia),

J.Ch. Moretti (IRAA-MOM, Université de Lyon 2/CNRS),

D. Raue (Ägyptisches Museum der Universität Leipzig), P. Reynolds (ICREA, Barcelona),

D. Welsby (British Museum, London)

\section{COMITÉ SCIENTIFIQUE DE LECTURE}

J. Holaubek (Institut für Ägyptologie, Wien), S. Ikram (AUC, Cairo),

K. Innemée (Universiteit Leiden), J. McKenzie (Faculty of Oriental Studies, University of Oxford),

N. Strudwick (University of Cambridge), A. Loprieno-Gnirs (Universität Basel),

Ch.E. Loeben (Museen für Kulturgeschichte, Hannover), Y. Tristant (Macquarie University, Sydney),

V.W.J. van Gerven Oei (University of Aberdeen), A. Peignard-Giros (HiSoMA-MOM, Université de Lyon 2/CNRS), J.A. Ostrowski, E. Papuci-Władyka, J. Śliwa (IA JU, Kraków), R. Czerner (WUST, Wrocław), A. Ćwiek (IA AMU, Poznań), M. Wiewióra (IA NCU, Toruń), K. Domżalski

(IAE PAS, Warszawa), K.O. Kuraszkiewicz (DE FOS UW), M. Barwik, P. Bieliński, P. Dyczek, W. Godlewski, D. Ławecka, S. Rzepka, J. Żelazowski, M. Gawlikowski, J. Młynarczyk, A. Niwiński, T. Sarnowski, D. Szeląg, T. Waliszewski (IA UW, Warszawa)

\section{RÉDACTEUR THÉMATIQUE DU VOLUME \\ Barbara Lichocka}

\author{
AIDE RÉDACTION TECHNIQUE \\ Dorota Dobrzyńska, Mariusz Drzewiecki
}

REVUE DES TEXTES ANGLAIS

Jo Harper 
ÉTUDES et TRAVAUX XXX 
INSTYTUT KULTUR ŚRÓDZIEMNOMORSKICH I ORIENTALNYCH POLSKIEJ AKADEMII NAUK

\section{STUDIA i PRACE}

XXX

\section{Ro IKŚSiO \\ ESA PAN}

WARSZAWA

2017 
INSTITUT DES CULTURES MÉDITERRANÉENNES ET ORIENTALES DE L'ACADÉMIE POLONAISE DES SCIENCES

\section{ÉTUDES et TRAVAUX}

XXX

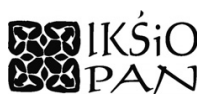

VARSOVIE

2017 
Publication scientifique financée dans le cadre du programme du Ministre de la Science et de l'Éducation Supérieure

« Programme National de Développement de l’Humanistique » pour les années 2016-2021 (projet no 3bH 150099 83)

\title{
HARODOWY PROGRAM ROZWOJU HUMANISTYKI
}

\author{
Copyright (C) \\ Instytut Kultur Śródziemnomorskich i Orientalnych PAN \\ et les Auteurs \\ Warszawa 2017
}

\author{
ISSN 2084-6762 \\ (avant $2011: 0079-3566$ ) \\ e-ISSN 2449-9579 \\ Version première en papier, imprimée en Pologne - 150 copies \\ Version électronique accessible sur \\ http://www.etudesettravaux.iksiopan.pl
}

Édition: Polskie Towarzystwo Historyczne et Wydawnictwo Neriton, Warszawa

Conception générale de couverture : J. Iwaszczuk

Photos de couverture : En haut, à gauche. Vieille Dongola 1991, S. Jakobielski

(debout à gauche), K. Pluskota (debout à droite), B. Żurawski (assis sur le camion)

et P. Wierzbicki (assis sur le camion) (de la collection de B. Żurawski)

En haut, à droite. Palmyre 1964, M. Marciniak au travail (phot. A. Dziewanowski)

Au centre. E. Laskowska-Kusztal au travail (de la collection de E. Laskowska-Kusztal)

En bas, à gauche. Tell Atrib 1962 ; de gauche : T. Biniewski, M. Marciniak, K. Kołodziejczyk,

K. Michałowski, A. Ostrasz, S. Jakobielski et S. Jasiewicz devant eux

(de la collection de IKŚSiO PAN).

En bas, à droite. Vieille Dongola 1976, S. Jakobielski nettoyant le mur (phot. M. Steinborn).

Au centre, à droite, K. Myśliwiec en train des travaux de documentation (de la collection de IKŚiO PAN) 


\section{Table des matières}

BARBARA LICHOCKA

Ergon agathon

Hartwig Altenmüller

$\mathrm{Zu}$ den Feindbildern auf den Zauberstäben des Mittleren Reiches und der Zweiten

Zwischenzeit

Nathalie Beaux

Des $m s w n s w$ de Thoutmosis III à Deir el-Bahari

Briant Bohleke, Nigel Strudwick

A Label for Opening of the Mouth Implements from the Burial of Senneferi (TT99)

and Remarks on the Ritual

Rosa Maria Bonacasa Carra, Nicola Bonacasa

Nuovi dati sugli edifici termali di Sabratha

EDWARD BROVARSKI

A Fragmentary Carrying Chair Scene in Salt Lake City, Utah

Julia Burdajewicz

Wall Painting Decoration from the North-West Church in Hippos-Sussita

of the Decapolis

Mariusz BURDAJEWICZ

From Pagan Temple to Church in Late Antiquity Palestine. A View from

Hippos-Sussita

MAREK ChlodNicki

Early Dynastic Bead Workshops at the Central Kom of Tell el-Farkha.

Patryk ChudziK, Mariusz Caban

Observations on the Architecture of the Tomb of Horhotep in Western Thebes

Krzysztof M. Cialowicz

New Discoveries at Tell el-Farkha and the Beginnings of the Egyptian State.

Amr EL-TiebI

Four Wooden New Kingdom Female Statuettes in the Egyptian Museum, Cairo 


\section{Naguib KanaWATI}

Ritual Marriage Alliances and Consolidation of Power in Middle Egypt during the Middle Kingdom

Adam Łajtar, Jolanta Mlynarczyk

A Faction Acclamation Incised on a Pithos Found Near the North-West Church at Hippos (Sussita)

Adam ŁaJTAR, Grzegorz OchaŁa

Two Private Prayers in Wall Inscriptions in the Faras Cathedral

Adam Łajtar, Anna Poludnikiewicz

Medicinal Vessels from Tell Atrib (Egypt)

JaCeK Michniewicz, Jolanta MlynarczyK

Petrographic Variability of the Fabrics of Wine Jars from Sha'ar-Ha Amakim as a Reflection of Differences in Their Provenance and Chronology

Iwona ModrzewsKa-PianetTI

Les importations d'amphores Dressel 20 en Gaule Cisalpine

Arthur SEgal

Samaria-Sebaste. Portrait of a polis in the Heart of Samaria 409

JOACHIM ŚLIWA

The Motif of a 'Blind Harper' in an Unexpected Place

MONIKA WIĘCH

Searching for the Kitchen in the Early Roman Phase of the 'Hellenistic' House at Nea Paphos (Cyprus)

Abréviations 
THE VOLUME IS PUBLISHED TO CELEBRATE

THE $60^{\text {TH }}$ ANNIVERSARY

OF THE ESTABLISHMENT OF

THE RESEARCH CENTRE FOR MEDITERRANEAN ARCHAEOLOGY POLISH ACADEMY OF SCIENCES

FOUNDED IN 1956

WHOSE MISSION IS CONTINUED BY

THE INSTITUTE OF MEDITERRANEAN AND ORIENTAL CULTURES

OF THE POLISH ACADEMY OF SCIENCES 